\title{
ROLE OF FILTERING TECHNIQUES IN COMPUTED TOMOGRAPHY (CT) IMAGE RECONSTRUCTION
}

\author{
Suheel Zargar ${ }^{1}$, Vaibhav Phad ${ }^{2}$, Praveen Kumar Poola ${ }^{3}$, Renu John ${ }^{4}$ \\ ${ }^{I}$ Department of Biomedical Engineering, Indian Institute of Technology, Hyderabad, Ordnance Factory Estate, \\ Yeddumailaram 502205, Telangana, India \\ ${ }^{2}$ Department of Biomedical Engineering, Indian Institute of Technology, Hyderabad, Ordnance Factory Estate, \\ Yeddumailaram 502205, Telangana, India \\ ${ }^{3}$ Department of Biomedical Engineering, Indian Institute of Technology, Hyderabad, Ordnance Factory Estate, \\ Yeddumailaram 502205, Telangana, India \\ ${ }^{4}$ Department of Biomedical Engineering, Indian Institute of Technology, Hyderabad, Ordnance Factory Estate, \\ Yeddumailaram 502205, Telangana, India
}

\begin{abstract}
With the arrival of Computed tomography $(C T)$ as a diagnostic tool in the field of medical imaging, the field of X-ray imaging got revolutionized. The CT technology processes various images which are taken at different angles in order to produce the crosssectional images of the specific part of the object with the help of a Computer. Post processing of the combinational images is done by the computer in offline. The CT Technology uses back projection process for recreating the images of the scanned object. The reconstructed object with simple projection technique looks blurry and cannot convey the information exactly, hence for better clarity Filtered back projection is done. In this paper we will discuss the various filtering techniques for better reconstruction of object.
\end{abstract}

Keywords: Computed Tomography, Sinogram, Sheep Logan Phantom, image reconstruction, filtered back projection, and image enhancement.

\section{INTRODUCTION}

We begin by explaining the process of CT reconstruction, working of scanner, and the process of image collection. In conventional CT machines the X-ray source and detectors rotate around the patient in a circular fashion thus collecting images of the object at various angles as shown in fig. 1 . The number of X-ray sources and the type of detection depends on the design of the CT machine. We know, that X-rays attenuate different materials differently depending on the material and its absorption coefficients [1]. That is the intensity of the X-ray beam keeps on decreasing as it penetrates further into the body. Every part of the body attenuates X-ray radiation differently depending on organ \& tissue density [2].

The intensity decreases exponentially with the distance traveled, and it can be understood from beer's lamberts law.

$$
I=I_{0} e^{-\alpha \mu}
$$

Where;

$\mu$ is the depth of penetration or thickness.

$\alpha$ is the characteristic feature and intrinsic property of the material which is known as its absorption coefficient.
The role of the detector here is to detect the amount of radiation that passes through the different organs and then convert the radiation intensities into the appropriate electrical signals. The electrical signals generated by this are then fed into the computer system which processes the data using various algorithms to generate the required images of the object.

In this article we begin with the CT introduction, in the second section we will explain the image reconstruction process, and in the third section we will explain about image acquisition, reconstruction process and how to remove the artifacts/noise present in the reconstructed images by various filtering techniques, and in the subsequent section we will analyze the results and then we will conclude. 


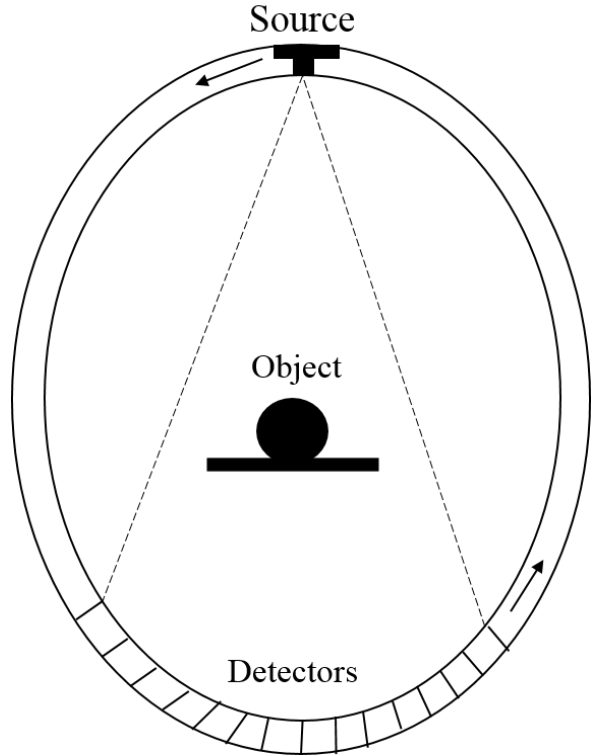

Fig -1: Configuration of conventional Third generation CT scanner.

\section{IMAGE ACQUISITION PROCESS}

Computer plays a key role in segmenting the organ/tissue into small volumes called voxels. Each Voxel from a scanned Body part is allocated a Number called as Attenuation Coefficient Number $\mu$ (mu). The X-ray transmission measurement $(\mathrm{Ni})$ represents the sum of all attenuation Values From all Voxels in each tissue slice. Each attenuation value will be expressed as the sum of voxel attenuation value along the radiation path. Bones have High $\mu$ values due to high attenuation and skin has low $\mu$ values. The measurements of the detector CT are proportional to the sum of attenuation coefficients [3].The pictorial representation of the detector with its measurements of $\mu$ values are shown in fig. 2 .

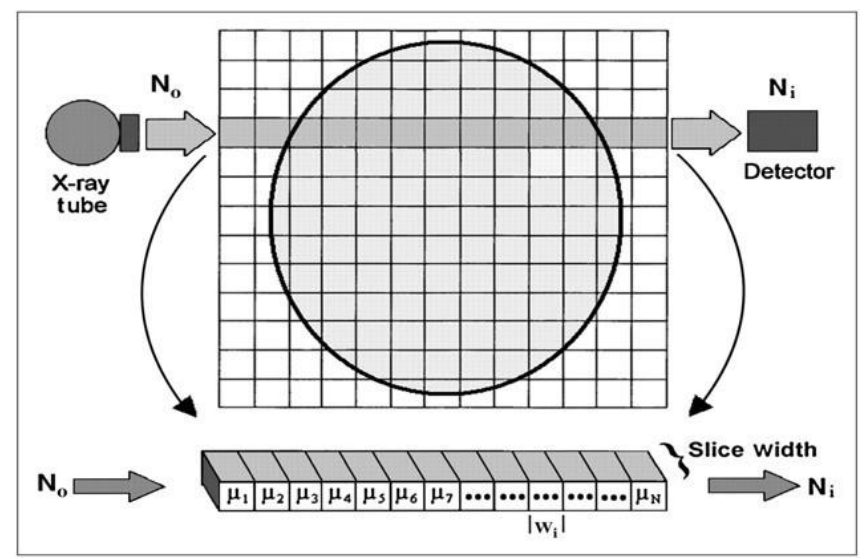

Fig -2: X-ray transmission measurement[3].

\subsection{Sinogram}

It's an Image of raw data acquired by CT scanner Before Reconstruction. The raw data which is acquired by the CT scanner for one CT slice can be displayed before reconstruction. This type of image is called the sinogram shown in fig.4 for our test object (Sheep Logan Phantom).
Sinograms are not useful for clinical proposes but the concepts are useful for tomographic principles. Rays are plotted vertically and views are shown horizontally in a sinogram. The position of a ray corresponding to the object varies sinosudially as a function of view angle. In our study we have taken $180^{\circ}$ angles for CT reconstruction of sheep Logan phantom shown in fig.3. Although the process runs for the entire $360^{\circ}$ but theoretically only $180^{\circ}$ parallel projections are necessary [3].

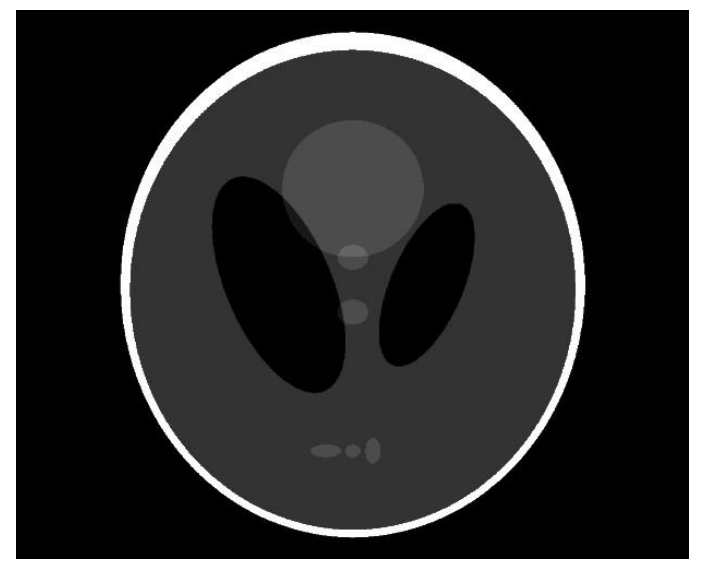

Fig -4: Sheep Logan Phantom (Test Image).

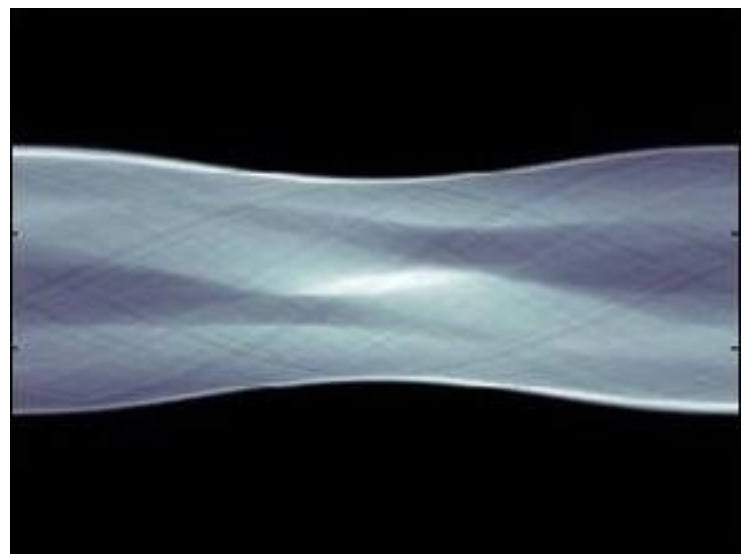

Fig -5: Sinogram of the sheep Logan Phantom.

\subsection{Back Projection}

Once the data is acquired by the CT Machine, we starts with an empty image matrix with all pixels set to zero. The attenuation value from each ray in all views is smeared or back projected onto image Matrix. The value of $\mu$ is added to each pixel in a line through the image corresponding to rays path as shown in fig.2. Image reconstruction is the process of estimating $2 \mathrm{D}$ object image slice $\mathrm{f}(\mathrm{x}, \mathrm{y})$ from a set of projections $\mathrm{p}(\mathrm{r}, \theta)$. The Back projection method to reconstruct an image is quite straight and less complicated, and performs in real time, but the image produced by back projection is quite blurry as shown in Fig.6. Projection Slice theorem tells us that if we have infinite number of 1-D projections of an object taken at infinite number of angles, we can reconstruct the original image by calculating its inverse radon transform [5]. 


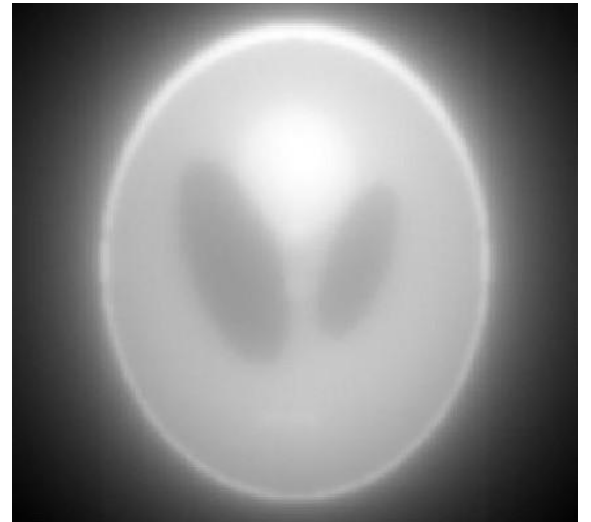

Fig -6: Image reconstructed using simple back projection Method.

\subsection{Simple Back Projection}

Simple back projection comes very close to desired CT image reconstruction. However, a characteristic 1/r Blurring is a by-product of simple back projection. In the case of simple back projection a single point on the image gets represented as a circular region whose intensity decreases as it moves away from the center. i.e., the point spread function of simple back projection is of a circularly symmetric, and increases as the function of its radius reciprocal, with the increase in radius the image blur gets increased.

\subsection{Filtered Back Projection}

Simple Back projection caused a Blurry image of the actual object. So in order to remove the $1 / \mathrm{r}$ dependency blurring and better the details of the scanned object we make use of the technique known as filtered back projection [4].In filtered Back projection the raw data are mathematically filtered before being projected onto the image matrix for the retrieval of scanned data. The blurring caused in the image can be eliminated by using the high pass filter [4]. The best way to eliminate such blurring is by using the ramp filter in combination with the High pass filter. Such a technique in which the simple back projection and ramp filtering are combined is known as filtered back projection and its reconstruction is shown in fig.7. In our study we have incorporated various filters which not only remove the blurring caused by the $1 / \mathrm{r}$ point spread function but also enhances the finest details of the scanned object and can be highly useful for the diagnostic purposes presenting a clear and highly preserved details of the object under investigation. In the coming sections we will discuss Methods of various filtering techniques along with their results and how they have been useful in making $\mathrm{CT}$ as a better diagnostic tool.

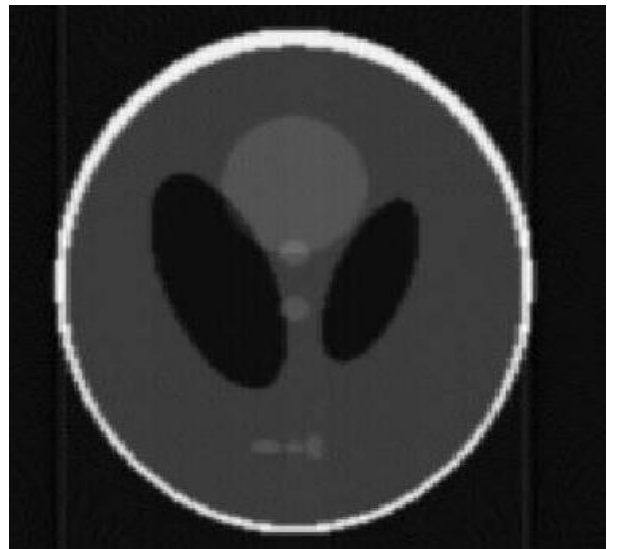

Fig -7: Back projection in combination with Ramp Filter. (Simple Filtered Back projection.)

\section{METHODS OF FILTERING TECHNIQUES FOR NOISE REDUCTION}

The filtered back projection is accomplished by combining the back projection with the Ramp filter. The blur caused in the unfiltered images is largely due to the low frequencies which are attenuated by the high pass filter thus aliasing the frequencies that cause the blur. The mathematical function of a Ramp Filter in frequency domain is given by:

$$
H_{R}\left(k_{x}, k_{y}\right)=k=\left(k_{x}^{2}+k_{y}^{2}\right)^{2},
$$

Where $k_{x}, k_{y}$ are spatial frequencies.

The Ramp is a compensatory filter as it eliminates the star artifact resulting from simple back projection. High pass filter sharpens the edges of the image (areas in an image where the signal changes rapidly) and increase the edge information of the object. The biggest drawback of using the high pass filter in this technique is it amplifies the statistical noises present in the image. In order to reduce the amplification of high-frequencies the ramp filter is always combined with a low-pass filter [6]. The usage of several combinations of such filters result in the better image reconstruction. The various filters that we have used along with their characteristic's and how they can provide better image reconstruction leading to better diagnostics in the CT imaging is discussed.

\subsection{Sheep-Logan Filter}

The filtered back projection image shown in the Fig 8 is the filtered image achieved by the Ram-Lak filter. The image produced by the Ram-Lak filter produces a noisy Image. Since the Ram-Lak filter is the High pass filter thus it causes the statistical noise in the image although it is able to remove the blurring. The sheep-Logan Filter which is similar to Lak filter has high influence in removing the High frequency Noise. The sheep Logan filter Multiplies the Ram-Lak filter with the Sinc filter. The Shepp-Logan filter belongs to the category of low pass filters which can be represented mathematically as: 


$$
S(f)=\frac{2 f_{m}}{\left[\pi\left(\sin |f| \pi / 2 f_{m}\right)\right]}
$$

The sheep Logan filter has a very high resolution and produces very least smoothing [7].

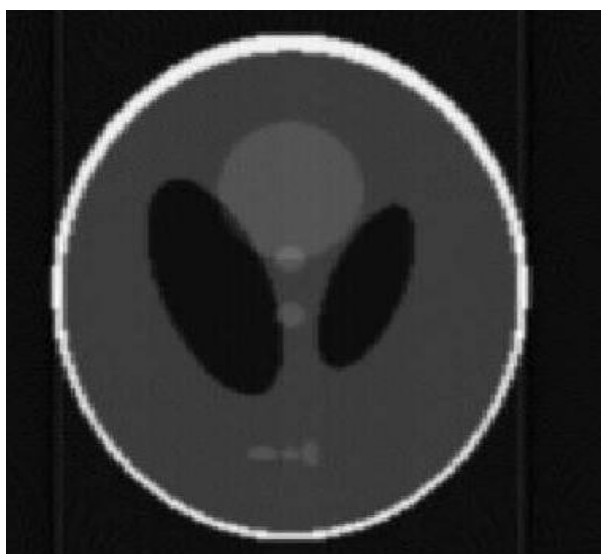

Fig -8: Image filtering using sheep Logan filter

\subsection{Hanning Filter}

The Hanning filter is also a low-pass filter whose characteristics are described by one parameter, the cut-off frequency. The Hanning filter is defined in the frequency domain as follows [8]:

$$
H(f)=\left\{\begin{array}{c}
0.50+0.50 \cos \left(\frac{\pi f}{f_{m}}\right), 0 \leq|f| \leq f_{m} \\
0, \text { otherwise }
\end{array}\right\}
$$

Here $\mathrm{f}$ are known as the spatial frequencies of the image and $f_{m}$ is the cut-off or critical frequency.

The Hann filter is highly effective in reducing image noise as it is reaching the zero quite quickly [9] as shown in fig.9. The one drawback of using Hann filter in the filtering is that it is not able to preserve the edges. We will look at various other filters who not only preserve edges but also enhance several other features of the image.

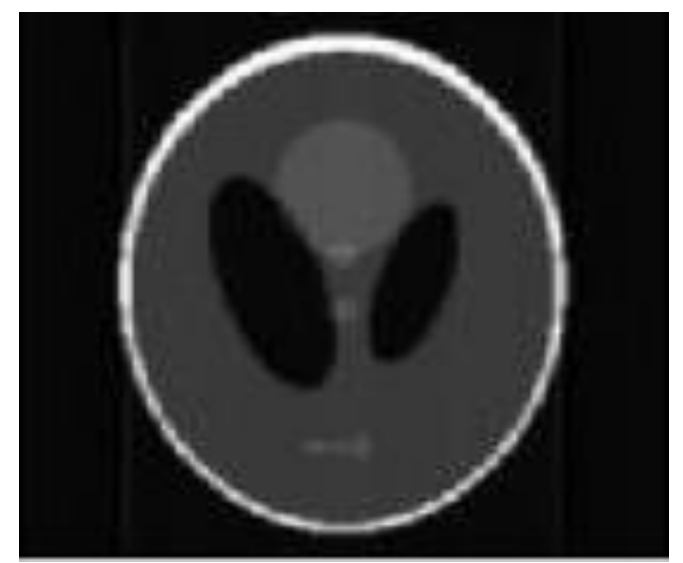

Fig -9: Image filtering using Hann Filter

\subsection{Hamming Filtering}

The Hamming filter is another kind of low pass filter, which is able to offer a high degree of smoothing, similar to the Hanning filter, the hamming filter also has only a single parameter to describe the shape of the filtering, which is like hanning filter called cut-off frequency. The mathematical representation of hamming filter is:

$$
H(f)=\left\{\begin{array}{c}
0.54+0.46 \cos \left(\frac{\pi f}{f_{m}}\right), 0 \leq|f| \leq f_{m} \\
\text { 0, otherwise }
\end{array}\right\}
$$

Where $\mathrm{f}$ are the spatial frequencies of the image and $f_{m}$ the cut off frequency.

The only difference between the hamming filter and the hanning filter is the amplitude at the cutoff frequency. The results achieved with the hamming filtering are shown in the Fig. 10.

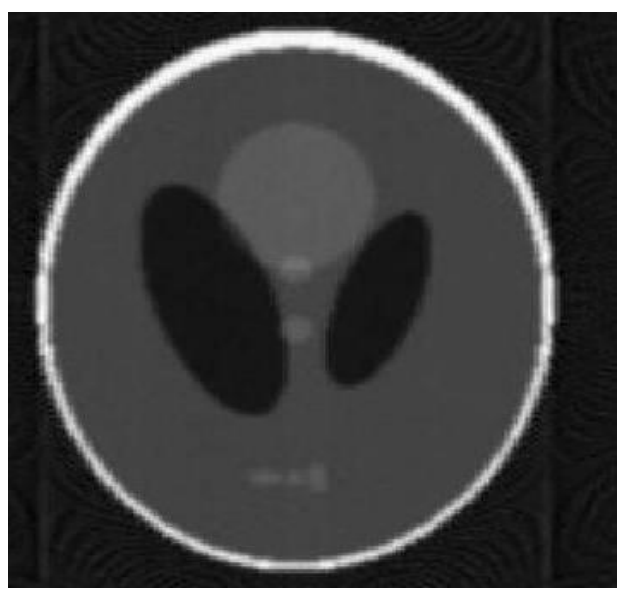

Fig -10: Image filtering using Hamming filter.

\subsection{Guided Image Filtering}

Guided image filtering helps in the removal of noises present in the image in a very efficient and better way. The filtering output of the guided filter is locally a linear transform of the guidance image. The guided filtering of the image results in the better edge-preserving and smoothing properties just like the bilateral filter, another advantage of guided filter is that such filtering is not affected by the gradient reversal artifacts. Also, the guided filter can be used beyond smoothing: by making use of the guidance image the filtering output can be made highly structured than the initial input [10]. The guided filter performs very well in a great variety of applications which including image smoothing/enhancement, HDR imaging, dehazing, and joint up sampling. Moreover, the guided filters are one of the fastest edge preserving filters thereby reducing a lot of computational time. We can see in fig.11, the difference in image reconstruction with the previous filtering techniques. 


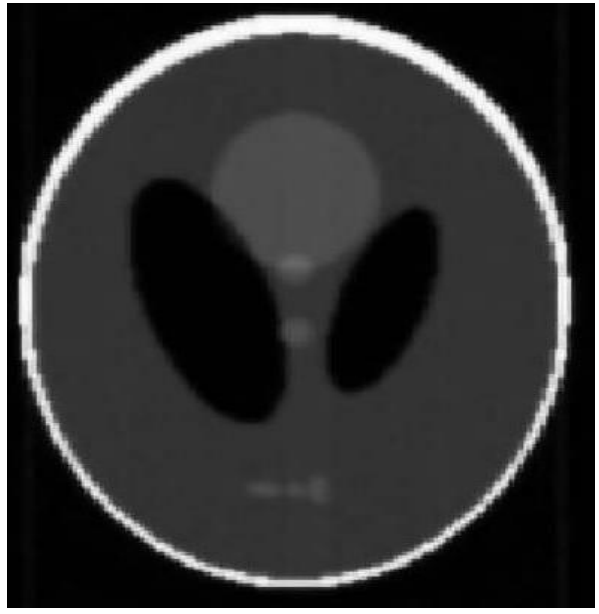

Fig -11: Image filtering using guided filter

\subsection{Median Filtering}

Median Filtering which is a nonlinear method and is highly useful in the removal of Salt and pepper noise.

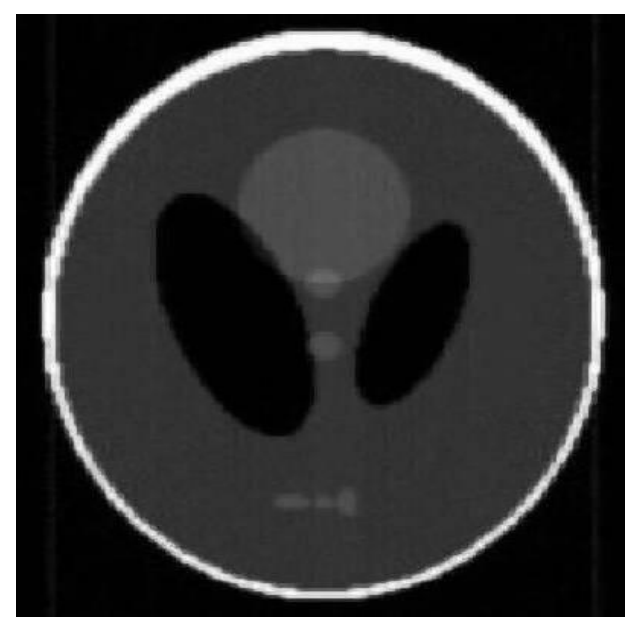

Fig -12: Image filtering using median filter

Salt and pepper noise is a very common noise among tomographic noises. In salt and pepper noise some of the pixels are corrupted thus occurring sparsely as black and white pixels. Such kind of noises can be removed by incorporating median filtering technique as shown in fig.12, which is a superior technique in comparison to the traditional mean filtering.

\subsection{Neighbourhood Sliding Filtering}

This is a spatial filter and is highly useful in noise suppression in the reconstructed image. In this filtering process images are filtered by sliding neighbourhood processing in which a mask is slide across the image. The mask is a filtering kernel made up of weighting factors who's values depend on the design, which can either attenuates or accentuates the certain properties of the image that is being processed [12].The reconstructed image with this filter is shown in fig. 13.

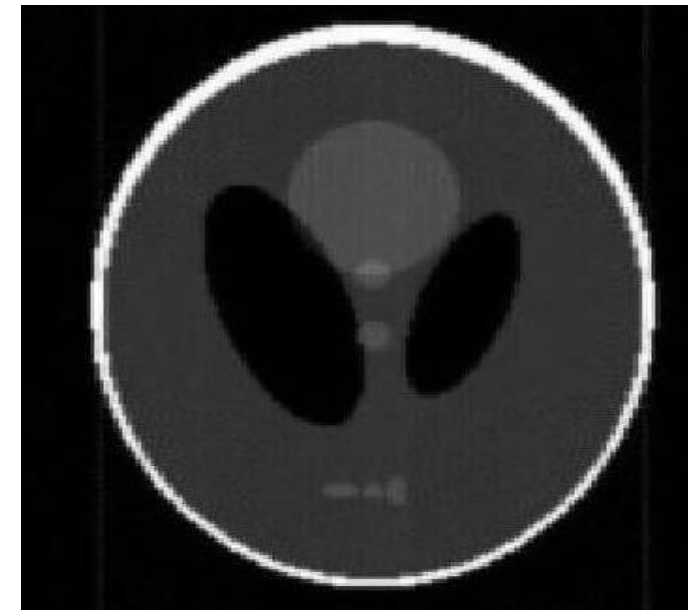

Fig -13: Image filtering using Neighbourhood sliding filter

All the reconstructed codes can be downloaded from the http://in.mathworks.com/matlabcentral/fileexchange/34608ct-reconstruction-package

Authors would like to acknowledge them for making the codes open source.

\section{CONCLUSION}

We saw the method of Back projection along with filtered back projection. We discussed how the blurring caused in the simple back projected image. We discussed in detail about the application of ramp filters (High pass filters). Followed by several low pass filters with the existing high pass filters combination, and we were able to see the reduction in noise caused by the low frequencies. Sheep Logan filter along with Hamming and hann filter proved beneficial to a large extend but still there existed the noises which were still unsuppressed. There were various kinds of noises present in the image thus reducing the details of the image. We also presented the filters like Guided filter and Neighbourhood sliding filter which were shown to increase the image enhancement. Such filters in addition to other discussed filters were shown to be very good at edge preservation and dehazing. They were seen to remove noises to a large extend. Guided filtering was shown to not suffer from reversal artifacts. The usage of these filters along with the commercially used filters can increase the image reconstruction efficiency to a large extend. The array based implementation of these filters will not only be computationally economical but also in terms of image preservation. Other advanced filters may also be used for image filtering. We leave this for future studies.

\section{REFERENCES}

[1]. Kalender, Willi A. "X-ray computed tomography." Physics in medicine and biology 51.13 (2006): R29.

[2]. McCullough, Edwin C., and J. Thomas Payne. "X-ray-transmission computed tomography." Medical physics 4.2 (1977): 85-98.

[3]. Hsieh, Jiang. "Computed tomography: principles, design, artifacts, and recent advances." Bellingham, WA: SPIE, 2009. 
[4]. Peters, Terry. "CT image reconstruction." SLIDES REVL (2002): 05697-05745.

[5]. Deans, Stanley R. The Radon transform and some of its applications. Courier Corporation, 2007.

[6]. Ziegler, A., Th Köhler, and R. Proksa. "Noise and resolution in images reconstructed with FBP and OSC algorithms for CT." Medical physics 34.2 (2007): 585-598.

[7]. Gui, Zhi-guo, and Yi Liu. "Noise reduction for low-dose X-ray computed tomography with fuzzy filter." OptikInternational Journal for Light and Electron Optics 123.13 (2012): 1207-1211.

[8]. Ahlstrom, M. L., and W. J. Tompkins. "Digital filters for real-time ECG signal processing using microprocessors." Biomedical Engineering, IEEE Transactions on 9 (1985): 708-713.

[9]. Riederer, Stephen J., Nobert J. Pelc, and David A. Chesler. "The noise power spectrum in computed $\mathrm{x}$-ray tomography." Physics in medicine and biology23.3 (1978): 446.

[10]. He, Kaiming, Jian Sun, and Xiaoou Tang. "Guided image filtering." Pattern Analysis and Machine Intelligence, IEEE Transactions on 35.6 (2013): 1397-1409.

[11]. Arias-Castro, Ery, and David L. Donoho. "Does median filtering truly preserve edges better than linear filtering?" The Annals of Statistics (2009): 1172-1206.

[12]. Lukac, Rastislav, et al. "Vector filtering for color imaging." Signal Processing Magazine, IEEE 22.1 (2005): 74-86.

[13]. Wang, Changhong, Taoyi Chen, and Zhenshen Qu. "A novel improved median filter for salt-and-pepper noise from highly corrupted images." Systems and Control in Aeronautics and Astronautics (ISSCAA), 2010 3rd International Symposium on. IEEE, 2010.

[14]. Lu, Ching-Ta, and Tzu-Chun Chou. "Denoising of saltand-pepper noise corrupted image using modified directional-weighted-median filter." Pattern Recognition Letters 33.10 (2012): 1287-1295. 